\title{
Computational Design and Experimental Validation of SAR Mixers
}

\author{
Md Readul Mahmud, Vladimir Viktorov and Carmen Visconte \\ DIMEAS, Politecnico di Torino, Corso Duca degli Abruzzi, 24-10129, Torino, Italy
}

\begin{abstract}
In this study, two novel split and recombine (SAR) 'H-C' and ' $\mathrm{Y}-\mathrm{Y}$ ' micromixers along with a known SAR 'Chain' mixer are presented. The efficiency and pressure drop at Reynolds numbers (Re) up to 100 were investigated numerically as well as experimentally. Numerical and experimental values of mixing efficiency and pressure drop coincide considerably, which validate the numerical model. Results show that the mixing efficiency of the Chain mixer is good only at $\mathrm{Re} \geq 50$ and the pressure drop is relatively high, whereas the $\mathrm{H}-\mathrm{C}$ and the $\mathrm{Y}-\mathrm{Y}$ mixers give a mixing efficiency higher than $90 \%$ over all the range of Reynolds numbers examined and their energy requirement is less than that of the Chain mixer. Furthermore, numerical residence time distribution (RTD) was explored, which successfully predicts the experimental results.
\end{abstract}

\section{Introduction}

Microfluidic devices have had a considerable impact in many fields such as biomedical diagnostics, drug development, and are widely used in the food and chemical industries [1]. Their function is to mix the reactants for initiation of the reaction process and they are an indispensable component in lab-on-chip (LOC) platforms for complex chemical reactions. Micromixers can also be integrated in a microfluidic system or work as stand-alone devices [2].

In design and evaluation of micromixers, the Reynolds number $\left(R e=\frac{\rho v d}{\mu}\right)$ is of major concern, where $\rho$ is the fluid density, $v$ is the velocity of fluid, $\mu$ is the dynamic viscosity, and $d$ is the characteristic length of the mixer. The characteristic dimension of a micromixer is in the sub-millimeter range and thus conventional methods to stir the fluids are not applicable [3]. Due to low Reynolds number, the flow in a microchannel is laminar and thus mixing is dominated by molecular diffusion rather than convection. Therefore, to get a good mixing performance, considerable time and long channel length are necessary, which yield high-pressure drop and high production cost [4]. Different kind of micromixers has been studied to overcome these problems. Micromixers can be classified into two categories, active and passive, depending on their mixing principle [5]. In active micromixers, active controls over the flow field are introduced to manipulate fluids to be mixed by using moving parts, acoustics waves or electro-magnetic fields or varying pressure gradient [6]. In the case of passive mixer, the mixing enhancement can be achieved by carefully designing the channel so as to create vortices and disturb the flow stability which, in turn, leads to the chaotic flow regime. As a consequence, the interfacial area among fluid segments is increased, reducing the diffusion length for heat and mass transport without any additional equipment or energy added to the system [7]. Passive micromixers are less expensive, easy to manufacture and maintain.

Good mixing performance and low pressure loss are two important design factors of any mixer. Low pressure drop is very crucial for industrial application. Good mixing efficiency is particularly suited for fast complex chemical reactions, where the reaction yields are strongly affected by mixing quality [7]. Degree of mixing can be achieved directly including experimentally [8] and numerically [9]; indirect method includes Poincare section analysis [10] and residence time distribution (RTD) [11]. Knowledge of the RTD enables the exact prediction of yield for first order reactions; the general reactor performance and mixing can be evaluated. Since micromixers are available in a great variety of geometries using different mixing principles, the selection of a suitable device for a given application becomes more challenging. Among various characteristics (like efficiency, energy loss) the knowledge of the RTD of a certain microfluidic device would be a useful help to identify the most appropriate one [12].

A number of experimental and numerical studies have been carried out on different split and recombine (SAR) mixers lately. SAR mixers show good mixing efficiency at low Reynolds number $(\operatorname{Re}<20)$ and high Reynolds number $(\operatorname{Re}>100)$, whereas mixing performance is relatively low at middle range [13]. Two novel SAR mixers, namely the $\mathrm{Y}-\mathrm{Y}$ and the $\mathrm{H}-\mathrm{C}$, are developed and studied at Reynolds numbers ranging from 1 to 100 . The proposed mixers show good efficiency (more than 90\%) and low pressure drop. Their performance is also compared to those of a known SAR mixer, the Chain one. 


\section{Micromixer design}

Two new passive SAR mixers, the $\mathrm{Y}-\mathrm{Y}$ and the $\mathrm{H}-\mathrm{C}$, are designed and constructed. Fig. 1 shows the fluid volume of the Chain, the $\mathrm{Y}-\mathrm{Y}$ and the $\mathrm{H}-\mathrm{C}$ micromixers with some important dimensions. As shown, all mixers are made up of four identical elements connected after one another, denoted as 1 to 4 . Micromixer geometries were micromilled in polycarbonate by a computer numerical control (CNC) Milling and Engraving Machine P20S, KUNLMANN (Germany) at I.T.D sas di Borgna Daniela e Borgna Eugenio \& C. (Turin, Italy).
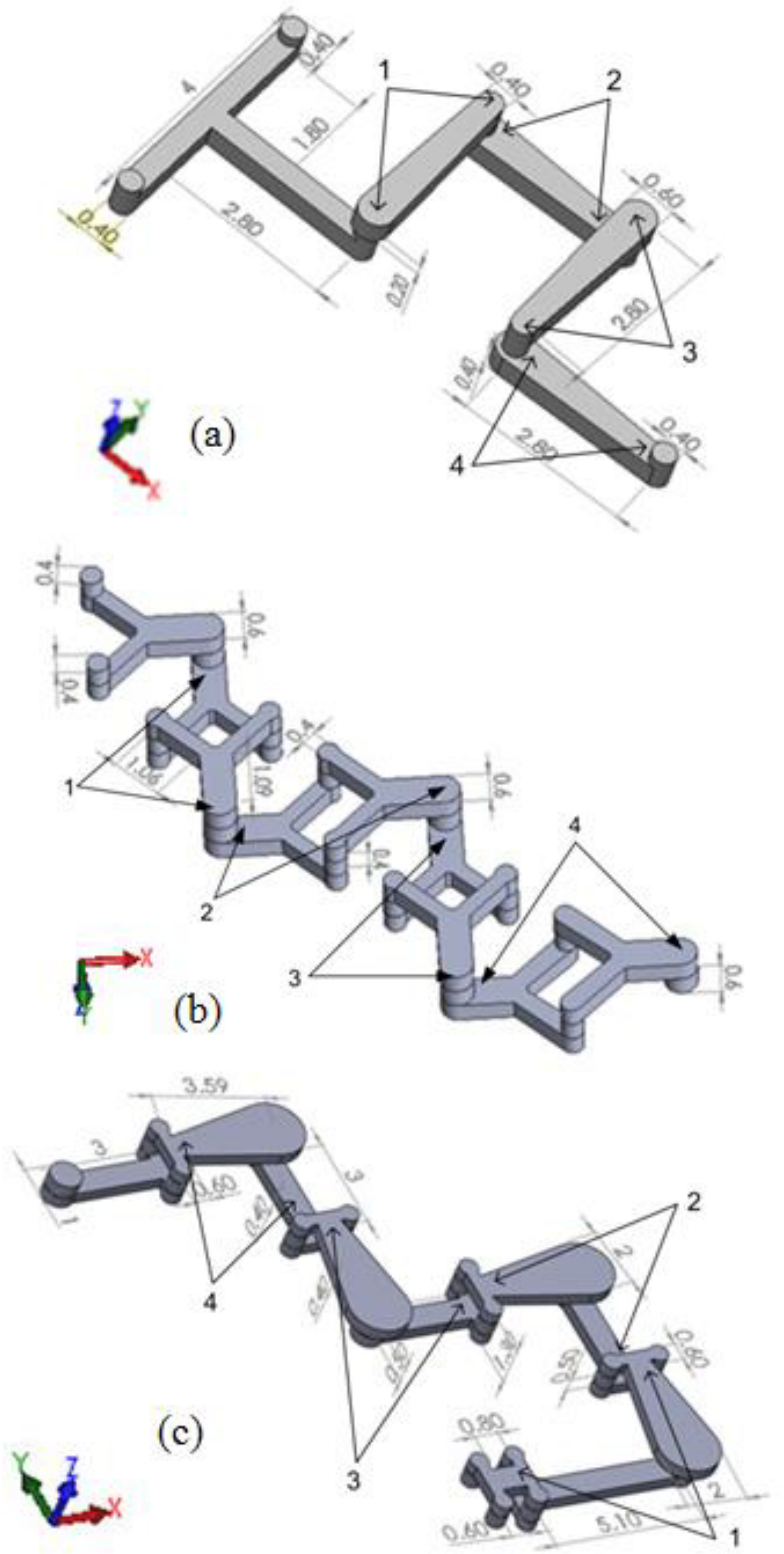

Figure 1. Geometry of (a) Chain, (b) Y-Y and (c) H-C micromixers.

\section{Mixing effectiveness}

To quantify the mixing performance the following equations were employed:

$$
\sigma=\sqrt{\frac{1}{N} \sum_{i=1}^{N}\left(C_{i}-C_{m}\right)^{2}}
$$

$$
\eta=1-\sqrt{\frac{\sigma^{2}}{\sigma_{\max }^{2}}}
$$

where $\sigma$ is the standard deviation of mass fraction; $N$ is the number of cells at a target area; $C_{i}$ is the mass fraction at a cell $i ; C_{m}$ is mean mass fraction at the target area; $\eta$ is the mixing efficiency; $\sigma_{\max }$ is the maximum standard deviation, namely 0.5 for symmetrical boundary conditions, used for normalization. The experimental efficiency and pressure drop were computed following the steps described in [13].

\section{Numerical methods}

\subsection{Numerical model of mixing}

The commercial CFD software Ansys Fluent 15 was employed for the numerical analysis. The fluid flow was assumed to be steady, laminar, Newtonian, and incompressible; therefore, ignoring the body force and gravity, governing equations are the continuity equation, Navier-Stokes equation and species convection-diffusion equation, given respectively by:

$$
\begin{gathered}
\nabla V=0 \\
\rho V \nabla V=-\nabla \mathrm{P}+\mu \nabla^{2} V \\
V \nabla C=\mathrm{D} \nabla^{2} C
\end{gathered}
$$

where $V$ is the fluid velocity vector, $\rho$ is the fluid density, $P$ is the pressure, $\mu$ is the fluid dynamic viscosity, $C$ is the species mass concentration, and $D$ is the diffusion coefficient of the species.

Water properties were applied to the mixing species: density and viscosity were set to constant values of 998 $\mathrm{kg} / \mathrm{m}^{3}$ and $1 \times 10^{-3} \mathrm{~kg} / \mathrm{m} \cdot \mathrm{s}$, respectively. The diffusion coefficient was set at $10^{-9} \mathrm{~m}^{2} / \mathrm{s}$, which is the typical value for an aqueous solution at room temperature. Both entrances were set to "velocity inlets"; the exit port was set to "pressure outlet", with a gauge pressure of $0 \mathrm{~Pa}$. At all walls no-slip boundary condition was applied.

Hexahedral cells were used to discretize the computational domain. Grid sensitivity test was performed for the mixing efficiency to determine the optimum number of grid points for all the micromixers. A grid system with approximately $3.53 \times 10^{5}, 5.12 \times 10^{5}$ and $5.25 \times 10^{5}$ nodes was chosen for the Chain, the Y-Y and the $\mathrm{H}-\mathrm{C}$ mixers, respectively.

\subsection{Numerical model of RTD}

The RTD is obtained commonly by injecting a tracer instantaneously (a pulse input) or at a constant rate (a step input) at the inlet of a flow system, and then measuring the tracer concentration at the outlet as a function of time. The RTD function, E(t), which describes quantitatively how much time different fluid elements have spent in a continuous flow system is defined mathematically so that $E(t) d t$ is the fraction of fluid elements that have spent a time between $t$ and $t+d t$ in the flow system [11]. In the 
determination of RTD in this study, the pulse input method is used in order to avoid the numerical errors from data differentiation that is inherent in the step input method. The time steps for each simulation are appropriately adjusted so as to limit the necessary number of calculation steps needed by the time-consuming transient simulations. For pulse tracer input method, E(t) is given by equation (6). When the mixing performance of flow systems of different sizes or flow conditions is to be compared, a normalized RTD function, E $(\theta)$, is used instead of $E(t)$. Both functions are related by equation 7 [14] where $t_{m}$ and $\theta$ are the mean residence time and dimensionless time, respectively.

$$
\begin{gathered}
E(t)=\frac{C(t)}{\int_{0}^{\infty} C(t) d t} \\
E(\theta)=t_{m} E(t) \\
t_{m}(t)=\frac{\int_{0}^{\infty} t C(t) d t}{\int_{0}^{\infty} C(t) d t} \\
\theta=\frac{t}{t_{m}}
\end{gathered}
$$

Carrying out the CFD simulations, the plots of E $(\theta)$ vs. $\theta$ are constructed for all the mixers. Some other important parameters, such as coefficient of variance $(\mathrm{CoV})$, variance $\left(\sigma(t)^{2}\right)$ and skewness (s), are defined by equations (10)-(12):

$$
\begin{gathered}
\operatorname{CoV}=\frac{\sigma(t)}{t_{m}} \\
\sigma(t)^{2}=\int_{0}^{\infty}\left(t_{i}-t_{m}\right)^{2} E(t) d t
\end{gathered}
$$

$$
s=\frac{\int_{0}^{\infty}\left(t_{i}-t_{m}\right)^{2} E(t) d t}{\sigma(t)^{3 / 2}}
$$

Once the RTD function is obtained, residence time, coefficient of variance, variance and skewness were calculated to quantify the degree or extent of mixing in continuous flow systems. The RTD of a microchannel mixer will deviate from that of an ideal plug flow mixer depending on the hydrodynamics within the microchannel. In the context of the static microchannel mixers designed to improve radial/transverse mixing, a $\mathrm{CoV}$ of zero would imply complete plug-flow mixing while a non-zero $\mathrm{CoV}$ implies that there is axial dispersion or mixing caused by non-uniform or laminar velocity profile and molecular diffusion. In this case, the smaller the variance or the $\mathrm{CoV}$, the narrower is the RTD, the closer is the distribution to the mean residence time, and the better the mixing quality [11], [15].

\section{Results and discussion}

The purpose of this study was to examine and compare the performance of three SAR micromixers.

Firstly, mixing efficiency of the Chain, the Y-Y and the $\mathrm{H}-\mathrm{C}$ mixers was evaluated experimentally and numerically over a range of Re numbers from 1 to 100 . Fig. 2 shows the concentration distribution of fluids along the length of mixers in both experimental tests and numerical simulations. As can be seen, a reasonable agreement between numerical and experimental results can be reported. However, there are still apparent differences in some sections. The main reason lies in the fact that simulation images can only show horizontal mid-plane. Therefore, it was not possible to take into consideration the multiple laminations of fluids layers. By contrast, the experimental images cover the whole depth of the channel viewable from below.
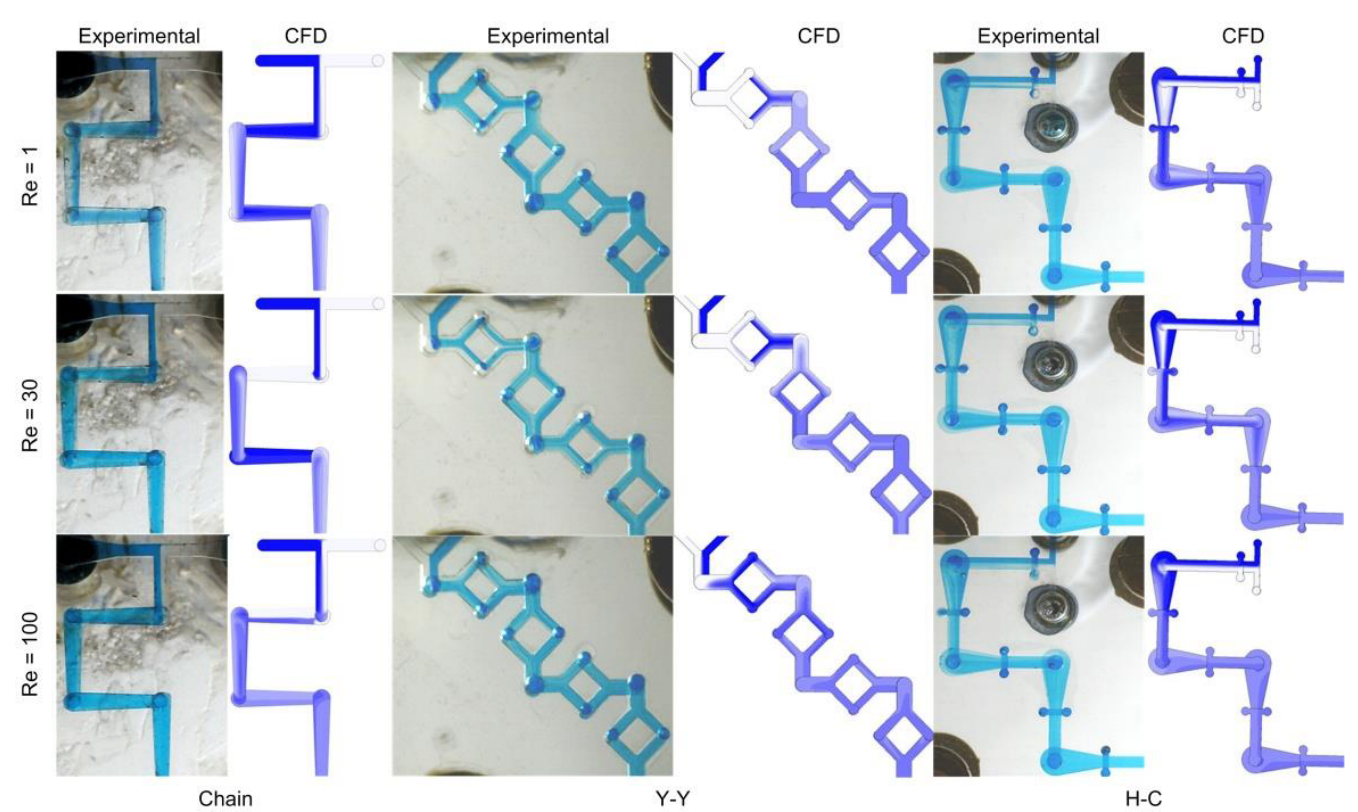

Figure 2. Comparison between the images taken from the numerical simulation and the experiments 


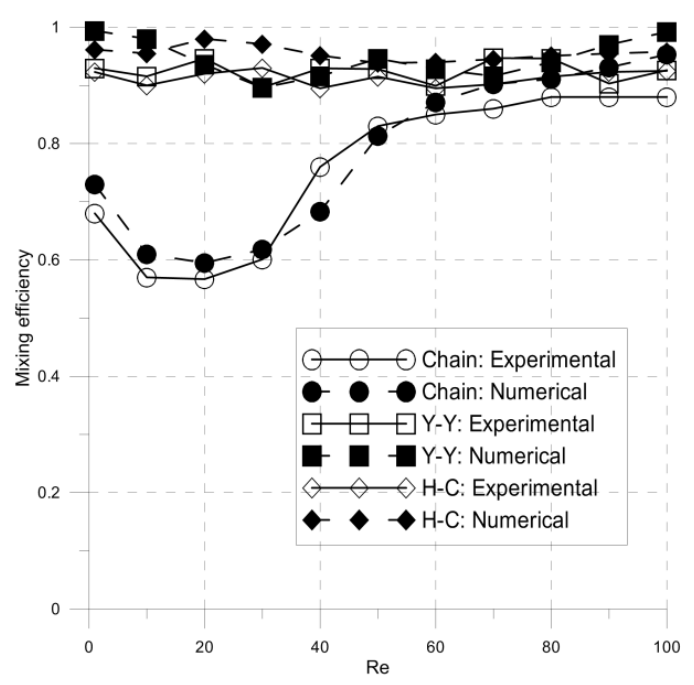

Figure 3. Experimental and numerical mixing efficiency

Figure 3 compares the mixing efficiency of the Chain, the $\mathrm{Y}-\mathrm{Y}$ and the $\mathrm{H}-\mathrm{C}$ mixers at the exit of the fourth element; as shown, experimental and numerical data have the same trend and reasonable agreement. The efficiency of the $\mathrm{Y}-\mathrm{Y}$ and the $\mathrm{H}-\mathrm{C}$ mixers does not depend on Reynolds numbers contrary to that of the Chain mixer. All three mixers show reasonable mixing efficiency at low Reynolds numbers due to the stratified flow. As the Reynolds numbers increase, there is a shorter residence time for the fluid particles to diffuse, and as a result worse mixing efficiency could be expected. Nevertheless, the Y-Y and the H-C mixers show good efficiency due to the three-dimensional change of direction of flow in the input of each element, which promotes a helical fluid motion; as a consequence, the fluid path is lengthened [13]. However, this swirling effect is less evident in the Chain mixer. Therefore, efficiency of the Chain mixer strongly depends on Reynolds numbers; in particular, at the low-middle range $(1 \leq \mathrm{Re} \leq 50)$ is less than $80 \%$. At high Reynolds numbers, the engulfment flow regime is reached and species get enough time to diffuse; therefore all mixers show an efficiency of about $90 \%$.

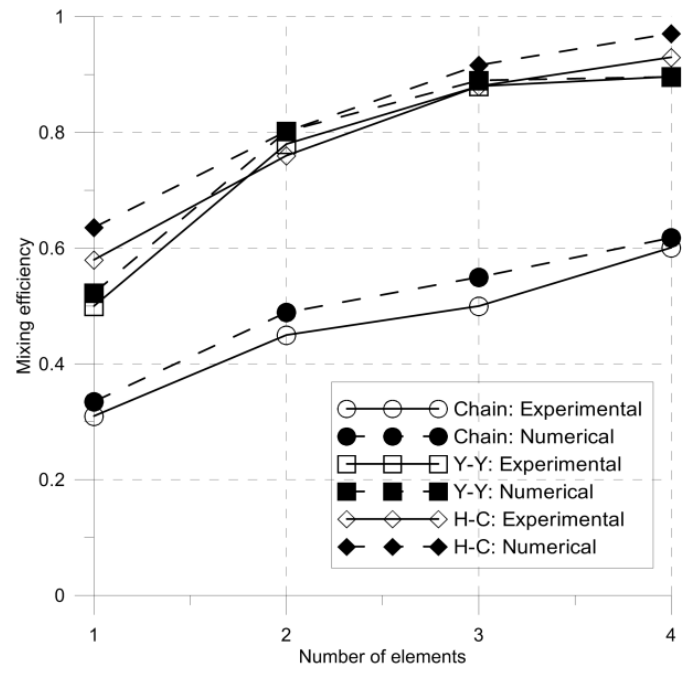

Figure 4. Mixing efficiency at $\mathrm{Re}=30$

Fig. 4 demonstrates the dependency of mixing efficiency on the number of elements at $\operatorname{Re}=30$.
Experimental and numerical efficiency curves have the same trend: mixing efficiency increases with the number of elements. It is clear that the efficiency of the Chain mixer is always less than those of the $\mathrm{Y}-\mathrm{Y}$ and the $\mathrm{H}-\mathrm{C}$ mixers; after the first and the third element the difference in efficiency is $10 \%$ and $30 \%$, respectively. Furthermore the Y-Y and H-C mixers show a mixing efficiency of almost $90 \%$ at the exit of the fourth element, whereas the Chain mixer shows an efficiency of only about $60 \%$.

Thirdly, the pressure drop of the micromixers was computed experimentally and numerically as shown in Fig. 5. Experimental and numerical pressure drop increases with the increase of flow rate, as expected. In addition, experimental and numerical values show satisfactory agreement. It can be concluded that the pressure drop is linearly dependent on the flow rate up to $2 \times 10^{-8} \mathrm{~m}^{3} / \mathrm{s}$ due to the multi-laminated flow of fluid. As the flow rate increases, vortexes are introduced in the fluid stream and consequently the pressure drop curve moves away from the linearity. The $\mathrm{H}-\mathrm{C}$ mixers show less pressure drop than other two mixers. Therefore, the $\mathrm{Y}-\mathrm{Y}$ and the $\mathrm{H}-\mathrm{C}$ mixers require less energy to operate at same flow condition.

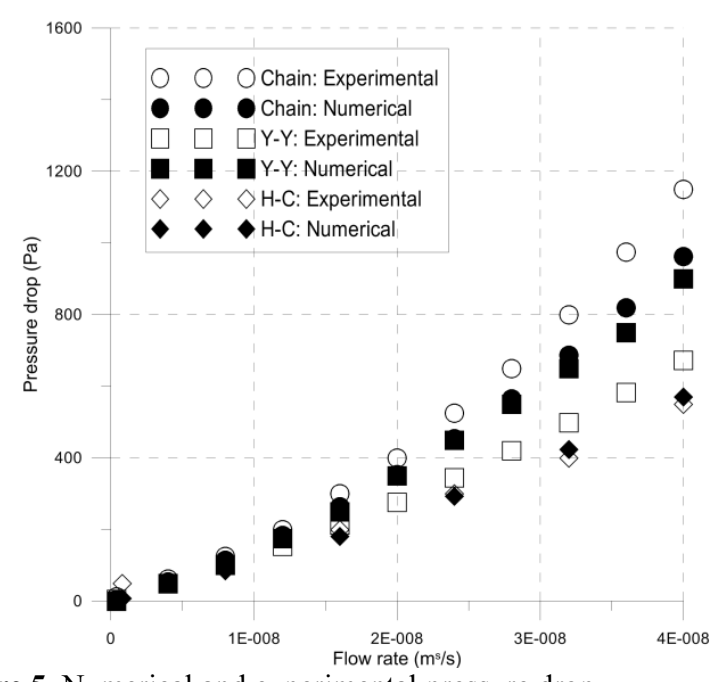

Figure 5. Numerical and experimental pressure drop

Table 1. Results of the numerical RTD analysis for at different Reynolds numbers

\begin{tabular}{|c|c|c|c|c|c|}
\hline \multicolumn{2}{|c|}{$\operatorname{Re}$} & $\begin{array}{c}\text { Residence } \\
\text { time (s) }\end{array}$ & $\mathrm{CoV}$ & Variance & Skewness \\
\hline \multirow{4}{*}{ 胥 } & 1 & 16.656 & 0.318 & 44.046 & 34.548 \\
\hline & 10 & 1.770 & 0.388 & 0.849 & 2.592 \\
\hline & 30 & 0.516 & 0.371 & 0.752 & 2.325 \\
\hline & 100 & 0.137 & 0.225 & 0.002 & 0.029 \\
\hline \multirow{4}{*}{$\stackrel{\text { U }}{1}$} & 1 & 41.897 & 0.259 & 204.329 & 151.876 \\
\hline & 10 & 4.293 & 0.266 & 2.773 & 8.643 \\
\hline & 30 & 1.331 & 0.192 & 0.128 & 0.707 \\
\hline & 100 & 0.421 & 0.153 & 0.007 & 0.063 \\
\hline \multirow{4}{*}{ خ } & 1 & 32.142 & 0.319 & 186.393 & 132.367 \\
\hline & 10 & 3.252 & 0.327 & 1.952 & 4.064 \\
\hline & 30 & 1.056 & 0.308 & 0.189 & 0.782 \\
\hline & 100 & 0.297 & 0.196 & 0.006 & 0.051 \\
\hline
\end{tabular}


Finally, using the results from the CFD simulations, the plots of $\mathrm{E}(\theta)$ vs. $\theta$ were constructed at different Reynolds number as shown in Fig. 6. Generally, all micromixers show similar behavior. At low Reynolds numbers the distributions are broader and at higher Reynolds numbers the RTD curves become narrower and more symmetric. A curve with a sharp peak or narrower peak, which also gives low value of $\mathrm{CoV}$, denotes better mixing efficiency. It is notable from Table 1 that the values of $\mathrm{CoV}$ and variance decrease with the increase of Reynolds numbers for both the Y-Y and the H-C mixers, which indicates the generation of secondary flow due to the helical fluid motion; as a result radial mixing have effect on total mixing performance. On the contrary in the case of the Chain mixer at $\mathrm{Re}=30$, the value of $\mathrm{CoV}$ is relatively high, which predicts a low mixing efficiency, as shown in Fig. 3. In addition, the residence time of the $\mathrm{Y}-\mathrm{Y}$ and the $\mathrm{H}-\mathrm{C}$ mixers is higher than that of the Chain mixer due to their longer channel length.
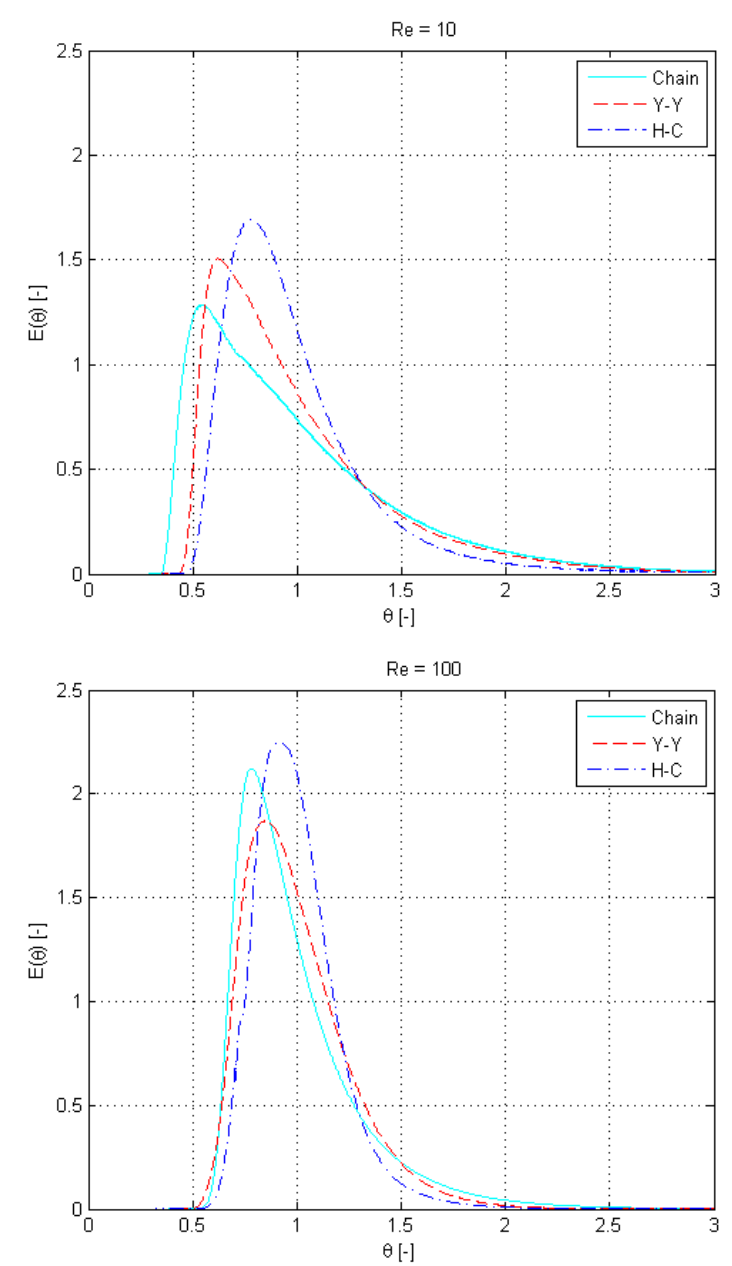

Figure 6. Normalized RTD of mixers at $\mathrm{Re}=10$ and 100

\section{Conclusions}

Three passive SAR micromixers, the Chain, the Y-Y and the $\mathrm{H}-\mathrm{C}$, were studied numerically at Reynolds numbers ranging from 1 to 100 . Afterwards, experiments were done to validate the numerical work. All micromixers are made up of four identical elements connected one after another. The mixer structures are simple and no grooves are provided. An image analysis technique was used to evaluate mixture homogeneity at four target areas along the mixer. A good agreement is found between numerical and experimental results. The Y-Y and the $\mathrm{H}-\mathrm{C}$ micromixers show an almost flat mixing characteristic over the examined range of Reynolds numbers, with a mixing efficiency higher than $90 \%$; conversely, the Chain mixer shows a relevant decrease of efficiency at low-mid range. In addition, pressure drop within the $\mathrm{Y}-\mathrm{Y}$ and the $\mathrm{H}-\mathrm{C}$ micromixers is less than that of the Chain mixer. Furthermore, RTD is analyzed at low, middle and high Reynolds number, which also predicts the better performance of the $\mathrm{Y}-\mathrm{Y}$ and the $\mathrm{H}-\mathrm{C}$ mixers than the Chain one. Therefore, the new Y-Y and H-C micromixers have high potential for chemical and food applications.

\section{References}

1. C. Lee, W. Wang, L. Fu, Micromachines 6, 1-24 (2015)

2. I. Sabotin, G. Tristo, M. Junkar, J. Valentincic, ChemE 91, 778-788 (2013)

3. A. A. Mouza, C. M. Patsa, F. Schonfeld, ChemE 86, 1128-1134 (2008)

4. A. Alam, A. Afzal, K. Kim, ChemE 92, 423-434 (2014)

5. S. Hossain, K. Kim, Micromachines 5, 913-928 (2014)

6. T. G. Kang, P. D. Anderson, Micromachines 5, 1270-1286 (2014)

7. N. Solehati, J. Bae, A. P. Sasmito, Computers \& Fluids 96,10-19 (2014)

8. Y. Liu, Y. Deng, P. Zhang, Z. Liu, J. Micromech. Microeng. 23, 1-10 (2003)

9. Y. Du, Z. Zhang, C. Yim, M. Lin, X. Cao, Micromachines 1, 19-33 (2010)

10. D. J. Beebe, R. J. Adrian, G. O. Olsen, M. A. Stremler, H. Aref, B. Jo, Mechanics \& Industries 2, 343-348 (2001)

11. J. T. Adeosun, A. Lawal, Chemical Engineering Science 64, 2422-2432 (2009)

12. D. Boskovic, S. Loebbecke, Chemical Engineering Journal 135S, S138-S146 (2008)

13. V. Viktorov M. R. Mahmud, C. Visconte, Micromachines 6, 1166-1179 (2015)

14. A. G. Kanaris, A. A. Mouza, Chemical Engineering Science 66, 5366-5373 (2011)

15. J. T. Adeosun, A. Lawal, Chemical Engineering Science 65, 1865-1874 (2010) 